\title{
Concavity Properties of Krein`s Spectral Shift Function
}

\author{
R. Geisler, V. Kostrykin \\ R. Schrader
}

SFB 288 Preprint No. 100

Diese Arbeit ist mit Unterstuitzung des von der Deutschen Forschungsgemeinschaft getragenen Sonderforschungsbereiches 288 entstanden und als Manuskript vervielfätigt worden.

Berlin, Dezember 1993 


\title{
CONCAVITY PROPERTIES OF KREIN'S SPECTRAL SHIFT FUNCTION
}

\author{
R. Geisler, V. Kostrykin*tand R. Schrader ${ }^{\ddagger}$ \\ Institut für Theoretische Physik \\ Freie Universität Berlin \\ Arnimallee 14, D-14195 Berlin, Germany
}

December 5, 1993

\begin{abstract}
We prove that the integrated Krein's spectral shift function for one particle Schrödinger operators in $\mathbf{R}^{3}$ is concave with respect to the perturbation potential. The proof is given by showing that the spectral shift function is the limit in the distributional sense of the difference of the counting functions for the given Hamiltonian and the free Hamiltonian in a finite domain $\Lambda$ with Dirichlet boundary conditions when $\Lambda \rightarrow \infty$.
\end{abstract}

\footnotetext{
*Supported by DFG, SFB 288 "Differentialgeometrie und Quantenphysik"

${ }^{\dagger}$ Permanent address: Department of Mathematical and Computational Physics, St. Petersburg State University, 198904 St. Petersburg, Russia

${ }^{\ddagger}$ E-mail: schrader@risc4.physik.fu-berlin.dbp.de
} 


\section{INTRODUCTION}

The spectral shift function for a pair of selfadjoint operators was introduced by Lifshitz [Lif] and Krein [Krl]. For given selfadjoint operators $H=H_{0}+$ $V$ and $H_{0}$ with suitable interaction potential $V$ the spectral shift function $\xi\left(\lambda ; H, H_{0}\right)$ satisfies

$$
\operatorname{tr}\left(\phi(H)-\phi\left(H_{0}\right)\right)=\int_{\mathbf{R}} \phi^{\prime}(\lambda) \xi\left(\lambda ; H, H_{0}\right) d \lambda
$$

for arbitrary $\phi \in C_{0}^{\infty}(\mathbf{R})$. This spectral shift function is related to scattering theory by which it essentially may be identified with the total phase shift $\delta$ at energy $\lambda>0$, i.e. $-\pi \xi\left(\lambda ; H, H_{0}\right)=\delta\left(\lambda ; H, H_{0}\right)$ [BiKr]. For recent review of the theory of the spectral shift function see [BiYa].

It was stated in [Kr2] (see also [BiYa]) that under certain normalizability conditions $\pm \xi\left(\lambda ; H_{0}+V, H_{0}\right) \geq 0$ almost everywhere provided that $\pm V \geq 0$. Later Kato [Ka] proved that the total phase shift is monotonic with respect to the perturbation potential, i.e.

$$
\delta\left(\lambda ; H_{0}+V_{1}, H_{0}\right) \geq \delta\left(\lambda ; H_{0}+V_{2}, H_{0}\right), \lambda \geq 0
$$

when $V_{1} \leq V_{2}$ provided that $\delta$ is chosen such that $\delta\left(\lambda ; H_{0}, H_{0}\right) \equiv 0$ and $\delta\left(\lambda ; H_{0}+V, H_{0}\right)$ is jointly continuous with respect to $V$ (in the operator norm sense) and $\lambda$.

In what follows we imply that the spectral shift function is normalized by the condition that $\xi\left(\lambda ; H_{0}+V, H_{0}\right)$ is identically zero for $\lambda$ below the spectra of $H_{0}+V$ and $H_{0}$. Under this normalization the inequality (1.1) implies that

$$
\xi\left(\lambda ; H_{0}+V_{1}, H_{0}\right) \leq \xi\left(\lambda ; H_{0}+V_{2}, H_{0}\right)
$$

for all $\lambda \in \mathbf{R}$ when $V_{1} \leq V_{2}$.

The aim of the present paper is to prove that the integrated spectral shift function

$$
\begin{array}{r}
\zeta\left(\lambda ; H, H_{0}\right)=\int_{-\infty}^{\lambda} \xi\left(\lambda^{\prime} ; H, H_{0}\right) d \lambda^{\prime}, \\
H=H_{0}+V, H_{0}=-\Delta
\end{array}
$$

is concave with respect to the perturbation potential V. Here and in what follows we will always work in $\mathbf{R}^{3}$, although an extension of our results to 
We mention also one trivial consequence of (1.3) for $\alpha=1 / 2$ and $V_{1}=$ $-V_{2}=V$ or (1.5) for $\alpha_{1}=\alpha_{2}=1$,

$$
\zeta\left(\lambda ; H_{0}+V, H_{0}\right)+\zeta\left(\lambda ; H_{0}-V, H_{0}\right) \leq 0 .
$$

It is worthwile to note that there is a close relationship between concave and subadditive functions of a single variable (see e.g. [Hi]). In particular, a necessary and sufficient condition that a measurable concave function $f(t)$ be subadditive in $\mathbf{R}_{+}$is that $f(+0) \geq 0$.

For the Laplace transform of $\xi\left(\lambda ; H, H_{0}\right)$ one can actually prove more. Denote

$$
F\left(t ; H, H_{0}\right)=\int_{\mathbf{R}} e^{-\lambda t} d \zeta\left(\lambda ; H, H_{0}\right) .
$$

Theorem 1.3 For any $0 \leq \alpha \leq 1$ and $V_{1}, V_{2} \in l^{1}\left(L^{2}\right)$

$$
\begin{array}{r}
F\left(t ; H_{0}+\alpha V_{1}+(1-\alpha) V_{2}, H_{0}\right) \geq \\
\alpha F\left(t ; H_{0}+V_{1}, H_{0}\right)+(1-\alpha) F\left(t ; H_{0}+V_{2}, H_{0}\right) .
\end{array}
$$

If in addition $V_{1}$ and $V_{2}$ are both nonnegative or both nonpositive, then

$$
\begin{aligned}
& F\left(t ; H_{0}+V_{1}+V_{2}, H_{0}\right) \leq F\left(t ; H_{0}+V_{1}, H_{0}\right)+F\left(t ; H_{0}+V_{2}, H_{0}\right), \\
& F\left(t ; H_{0}+V_{1}-V_{2}, H_{0}\right) \geq F\left(t ; H_{0}+V_{1}, H_{0}\right)+F\left(t ; H_{0}-V_{2}, H_{0}\right) .
\end{aligned}
$$

Comparing the claims of Theorems 1.1 and 1.3 it is natural to ask the question whether (1.4) and (1.5) can be generalized to

$$
\begin{gathered}
\zeta\left(\lambda ; H_{0}+V_{1}+V_{2}, H_{0}\right) \leq \zeta\left(\lambda ; H_{0}+V_{1}, H_{0}\right)+\zeta\left(\lambda ; H_{0}+V_{2}, H_{0}\right) \\
\zeta\left(\lambda ; H_{0}+V_{1}-V_{2}, H_{0}\right) \geq \zeta\left(\lambda ; H_{0}+V_{1}, H_{0}\right)+\zeta\left(\lambda ; H_{0}-V_{2}, H_{0}\right)
\end{gathered}
$$

whenever $V_{1}$ and $V_{2}$ are either both nonnegative or both nonpositive. In the general case the answer is negative. In the Appendix relation (1.9) is shown not to hold for the special case that $V_{1}$ and $V_{2}$ are point interactions centered at different points.

We note that the Laplace transform respects the canonical ordering for real valued functions on $\mathbf{R}$ in the sense that $f(\lambda) \geq 0$ implies $\int e^{-\lambda t} f(\lambda) d \lambda \geq$ 
For such operators the inequalities (1.3) and (1.4) follow from certain properties of the eigenvalues for the Dirichlet problem. We then consider the limit when the size of the domain $\Lambda$ tends to infinity. The inequalities (1.3) and (1.4) for the whole space are then proved by means of limiting arguments. In this context we would like to emphasize that the spectral shift function is a natural object for studying the relations between the spectral characteristics of the Hamiltonians in a whole space and in a finite domain. Physical arguments for the validity of these relations have been given previously in $[\mathrm{BaBl}]$.

\section{SCHRÖDINGER OPERATORS IN A FINITE VOLUME}

Let $\Lambda$ be a bounded open subset of $\mathbf{R}^{3}$. Let $H_{0, \Lambda}$ denote the Friedrichs extension of $-\Delta$ on $C_{0}^{\infty}(\Lambda)$, i.e. $H_{0, \Lambda}$ is the Dirichlet Laplacian for the domain $\Lambda$. Let $V \in \mathcal{K}$, where $\mathcal{K}$ is the Kato class, i.e. the set of all realvalued measurable functions in $\mathbf{R}^{3}$ satisfying

$$
\lim _{a \rightarrow 0} \sup _{x \in \mathbf{R}^{3}} \int_{|x-y| \leq a} \frac{|V(y)|}{|x-y|} d y=0 .
$$

To abbreviate notations, we also denote by $V$ the restriction of $V$ to $\Lambda$, considered as a multiplication operator in $L^{2}(\Lambda, d x)$. It is known (see e.g. [CaLa]) that $V$ is $H_{0, \Lambda}$-form bounded with relative bound 0 , so that by the KLMN theorem (see e.g. [RS4]) $H_{\Lambda}=H_{0, \Lambda}+V$ is selfadjoint on the domain $\mathcal{Q}\left(H_{\Lambda}\right)=\mathcal{Q}\left(H_{0, \Lambda}\right)=H_{0}^{1}(\Lambda) \subset L^{2}(\Lambda, d x)$, which is the completion of $C_{0}^{\infty}(\Lambda)$ in the norm $\|f\|_{H_{0}^{1}}=\left(\|f\|^{2}+\|\nabla f\|^{2}\right)^{1 / 2}$. Moreover, $H_{\Lambda}$ is semibounded and there is $b<\inf \operatorname{spec}\left(H_{\Lambda}\right)$ uniformly in $\Lambda$. It is well known (see e.g. [RS4]) that $\exp \left\{-t H_{0, \Lambda}\right\}$ and $\exp \left\{-t H_{\Lambda}\right\}$ are trace class for all $t>0$.

Let $N_{\Lambda}(\lambda ; V)$ be the counting function of the Hamiltonian $H_{\Lambda}=H_{0, \Lambda}+V$,

$$
N_{\Lambda}(\lambda ; V)=\sharp\left\{\mu \mid \mu<\lambda \text { is an eigenvalue of } H_{\Lambda}\right\} .
$$

Here and in what follows eigenvalues are counted with multiplicities. Let $N_{0, \Lambda}(\lambda)=N_{\Lambda}(\lambda ; V=0)$. 
We start the proof of this theorem by establishing some preparatory results. The following lemma is a well known consequence of the minimax principle (see e.g. [Th]).

Lemma 2.3 Let $H(\alpha)=H_{0}+\alpha V_{1}+(1-\alpha) V_{2}, \alpha \in[0,1]$ be selfadjoint operators and $\mathcal{Q}\left(V_{i}\right) \supset \mathcal{Q}\left(H_{0}\right), i=1,2$. Then the sum of the first $N$ eigenvalues $\lambda_{i}(\alpha)$ of $H(\alpha)$ is concave function in $\alpha$, i.e.

$$
\sum_{i=1}^{N} \lambda_{i}(\alpha) \geq \alpha \sum_{i=1}^{N} \lambda_{i}(1)+(1-\alpha) \sum_{i=1}^{N} \lambda_{i}(0)
$$

holds for any $\alpha \in[0,1]$.

Proof of Theorem 2.2 Let $\lambda_{i}(\alpha)$ be the $i$-th eigenvalue of the Hamiltonian $H_{0, \Lambda}+\alpha V_{1}+(1-\alpha) V_{2}$. First we show that the inequality

$$
\begin{array}{r}
\sum_{i=1}^{N_{\Lambda}\left(\lambda ; \alpha V_{1}+(1-\alpha) V_{2}\right)}\left(\lambda_{i}(\alpha)-\lambda\right) \geq \\
\alpha \sum_{i=1}^{N_{\Lambda}\left(\lambda ; V_{1}\right)}\left(\lambda_{i}(1)-\lambda\right)+(1-\alpha) \sum_{i=1}^{N_{\Lambda}\left(\lambda ; V_{2}\right)}\left(\lambda_{i}(0)-\lambda\right)
\end{array}
$$

holds for arbitrary $V_{1}, V_{2} \in \mathcal{K}, \lambda \in \mathbf{R}$ and $0 \leq \alpha \leq 1$. We fix the values of $\alpha$ and $\lambda$ and set $N=N_{\Lambda}\left(\lambda ; \alpha V_{1}+(1-\alpha) V_{2}\right)$. Then by Lemma 2.2

$$
\sum_{i=1}^{N}\left(\lambda_{i}(\alpha)-\lambda\right) \geq \alpha \sum_{i=1}^{N}\left(\lambda_{i}(1)-\lambda\right)+(1-\alpha) \sum_{i=1}^{N}\left(\lambda_{i}(0)-\lambda\right) .
$$

Note that if $N>N_{\Lambda}\left(\lambda ; V_{n}\right)$ for some $n=1,2$ then

$$
\sum_{i=N_{\Lambda}\left(\lambda ; V_{n}\right)+1}^{N}\left(\lambda_{i}(2-n)-\lambda\right) \geq 0
$$

and if $N<N_{\Lambda}\left(\lambda ; V_{n}\right)$ for some $n=1,2$ then

$$
\sum_{i=N+1}^{N_{\Lambda}\left(\lambda ; V_{n}\right)}\left(\lambda_{i}(2-n)-\lambda\right) \leq 0 .
$$


the leading terms of the asymptotic expansions of $\operatorname{tr} e^{-t\left(H_{0, \Lambda}+V\right)}$ and $\operatorname{tr} e^{-t H_{0, \Lambda}}$ for small $t$ have the same form and are determined by the geometry of the domain $\Lambda$. Thus they are canceled in the difference $\operatorname{tr} e^{-t\left(H_{0, \Lambda}+V\right)}-\operatorname{tr} e^{-t H_{0, \Lambda}}$.

Note also that the results similar to the statement of Lemma 2.3 for the case of the whole space $\left(\Lambda=\mathbf{R}^{3}\right)$ are well known (see e.g. [Bu, Sch, CdV, Ro] and references therein). We mention also the the paper [FOW] where the use of the Feynman-Kac formula has been proposed in order to obtain such kind of asymptotics.

Proof of Lemma 2.4 Consider

$$
\begin{array}{r}
F_{\Lambda}(t ; W)=\int_{\mathbf{R}} e^{-\lambda t} d \zeta\left(\lambda ; H_{0, \Lambda}+W+V, H_{0, \Lambda}+W\right)= \\
-\frac{1}{t} \operatorname{tr}\left(e^{-t\left(H_{0, \Lambda}+W+V\right)}-e^{-t\left(H_{0, \Lambda}+W\right)}\right) .
\end{array}
$$

We show that $F_{\Lambda}(t ; W)=(4 \pi t)^{-3 / 2} \int_{\Lambda} V(x) d x[1+o(1)]$ when $t \rightarrow+0$. Then the claim (2.7) of the lemma follows by Karamata's Tauberian theorem (see e.g. [Wi]). The claim (2.6) is then a particular case of $(2.7)$ when $W \equiv 0$.

We note that in the case of $\mathbf{R}^{3}$ we have the inclusion $l^{1}\left(L^{2}\right) \subset L^{1} \cap L^{2} \subset \mathcal{K}$. Since $V, W \in \mathcal{K}$ the operator $K_{\Lambda}(t)=\exp \left\{-t H_{0, \Lambda}+V+W\right\}-\exp \left\{-t H_{0, \Lambda}+\right.$ $W\}$ is an integral operator with jointly continuous bounded kernel $K_{\Lambda}(x, y ; t)$ and can be given by means of the Feynman-Kac formula [Si2]

$$
\begin{array}{r}
K_{\Lambda}(x, y ; t)=(4 \pi t)^{-3 / 2} \mathbf{E}_{0, x}^{t, y}\left\{\exp \left\{-\int_{0}^{t}\left(V\left(X_{s}\right)+W\left(X_{s}\right)\right) d s\right\}-\right. \\
\left.\exp \left\{-\int_{0}^{t} W\left(X_{s}\right) d s\right\} ; t<T_{\Lambda}\right\},
\end{array}
$$

where $\mathbf{E}_{0, x}^{t, y}$ stands for the conditional expectation with respect to the probability measure of the Brownian motion starting from $x$ at time 0 and conditioned to be at $y$ at time $t$. Also for the domain $\Lambda, T_{\Lambda}(\omega)$ is the first exit time for the Brownian path $\omega$, i.e.

$$
T_{\Lambda}(\omega)=\inf \left\{t>0, X_{t}(\omega) \notin \Lambda\right\},
$$

where $X_{t}(\omega)$ is the coordinate of $\omega$ at time $t$. Since $K_{\Lambda}(t)$ is trace class one can calculate its trace as the integral of $K_{\Lambda}(x, x ; t)$. Therefore one gets

$$
F_{\Lambda}(t ; W)=-\frac{1}{t}(4 \pi t)^{-3 / 2}
$$




$$
\left.\left[\exp \left\{-\int_{u}^{t} W\left(X_{s}\right) d s\right\}-1\right] ; t<T_{\Lambda}\right\} .
$$

Consider the first term $F_{\Lambda}^{(1)}(t)$. Since $V \in L^{1}$ we can apply Fubini's theorem to interchange the order of the integration. This yields

$$
\begin{aligned}
F_{\Lambda}^{(1)}(t)= & \frac{1}{t}(4 \pi t)^{-3 / 2} \int_{\Lambda} d x \int_{0}^{t} d u \mathbf{E}_{0, x}^{t, x}\left\{V\left(X_{u}\right) ; t<T_{\Lambda}\right\}= \\
& \frac{1}{t} \int_{\Lambda} d x \int_{0}^{t} d u \int_{\Lambda} d y P_{\Lambda}(x, y ; u) V(y) P_{\Lambda}(y, x ; t-u),
\end{aligned}
$$

where $P_{\Lambda}(x, y ; t)$ is the integral kernel of $\exp \left\{-t H_{0, \Lambda}\right\}$. By the semigroup property

$$
F_{\Lambda}^{(1)}(t)=\frac{1}{t} \int_{\Lambda} d x \int_{0}^{t} d u P_{\Lambda}(x, x ; t) V(x)=\int_{\Lambda} d x P_{\Lambda}(x, x ; t) V(x) .
$$

It is known that $P_{\Lambda}(x, x ; t) \rightarrow(4 \pi t)^{-3 / 2}$ as $t \rightarrow 0$ for almost all $x \in \Lambda$ and $P_{\Lambda}(x, x ; t) \leq P_{\Lambda=\mathbf{R}^{3}}(x, x ; t)=(4 \pi t)^{-3 / 2}$ (see e.g. [Sil]). Then by the dominated convergence theorem we get that

$$
(4 \pi t)^{3 / 2} F_{\Lambda}^{(1)}(t) \rightarrow \int_{\Lambda} V(x) d x
$$

when $t \rightarrow 0$.

We claim that $F_{\Lambda}^{(i)}(t ; W)=O\left(t^{-3 / 2+\epsilon}\right), i=2,3,4$ for some $\epsilon>0$ uniformly in $\Lambda$ when $t \rightarrow 0$.

Consider $F_{\Lambda}^{(2)}(t ; W)$. Obviously,

$$
\begin{array}{r}
\left|F_{\Lambda}^{(2)}(t ; W)\right| \leq \frac{(4 \pi t)^{-3 / 2}}{t} \int_{\Lambda} d x \mathbf{E}_{0, x}^{t, x}\left\{\int_{0}^{t} d u\left|W\left(X_{u}\right)\right|\right. \\
\cdot \mid \exp \left\{-\int_{u}^{t}\left(W\left(X_{s}\right)+V\left(X_{s}\right)\right) d s\right\}- \\
\left.\exp \left\{-\int_{u}^{t} W\left(X_{s}\right) d s\right\} \mid ; t<T_{\Lambda}\right\} \leq \\
\frac{(4 \pi t)^{-3 / 2}}{t} \int_{\mathbf{R}^{3}} d x \mathbf{E}_{0, x}^{t, x}\left\{\int_{0}^{t} d u\left|W\left(X_{u}\right)\right| \exp \left\{-\int_{u}^{t} W\left(X_{s}\right) d s\right\} .\right. \\
\left.\cdot\left|\exp \left\{-\int_{u}^{t} V\left(X_{s}\right) d s\right\}-1\right|\right\} .
\end{array}
$$


We carry out the integration with respect to $u$ explicitly. Corresponding calculations can be found e.g. in [Le, p.221] and we only give the final result

$$
(4 \pi t)^{3 / 2} \int_{0}^{t} d u P(x, y ; t-u) P(y, x ; u)=\frac{1}{2 \pi} \frac{e^{-\frac{|x-y|^{2}}{t}}}{|x-y|} .
$$

Therefore

$$
\begin{array}{r}
\int_{\mathbf{R}^{3}} d x \mathbf{E}_{0, x}^{t, x}\left\{\left(\int_{0}^{t} d u\left|W\left(X_{u}\right)\right|\right)^{p}\right\}^{1 / p} \leq \\
\frac{t^{(p-1) / p}}{(2 \pi)^{1 / p}} \int_{\mathbf{R}^{3}} d x\left(\int_{\mathbf{R}^{3}} d y \frac{e^{-\frac{|x-y|^{2}}{t}}}{|x-y|}|W(y)|^{p}\right)^{1 / p} .
\end{array}
$$

Recall now that $\Delta_{j}$ denotes the unit cube centered at the point $j \in \mathbf{Z}^{3}$. Since $(a+b)^{1 / p} \leq a^{1 / p}+b^{1 / p}$ for $a, b \geq 0$ and $p>1$, we get

r.h.s. of $(2.10)=$

$$
\begin{gathered}
\frac{t^{(p-1) / p}}{(2 \pi)^{1 / p}} \int_{\mathbf{R}^{3}} d x\left(\sum_{j \in \mathbf{Z}^{3}} \int_{\Delta_{j}} d y \frac{e^{-\frac{|x-y|^{2}}{t}}}{|x-y|}|W(y)|^{p}\right)^{1 / p} \leq \\
\frac{t^{(p-1) / p}}{(2 \pi)^{1 / p}} \int_{\mathbf{R}^{3}} d x \sum_{j \in \mathbf{Z}^{3}}\left(\int_{\Delta_{j}} d y \frac{e^{-\frac{|x-y|^{2}}{i}}}{|x-y|}|W(y)|^{p}\right)^{1 / p} .
\end{gathered}
$$

By the Hölder inequality and translation invariance of the Lebesgue measure

$$
\begin{gathered}
\text { r.h.s. of }(2.10) \leq \\
\frac{t^{(p-1) / p}}{(2 \pi)^{1 / p}} \int_{\mathbf{R}^{3}} d x \sum_{j \in \mathbf{Z}^{3}}\left(\int_{\Delta_{j}} \frac{e^{-\gamma \frac{|x-y|^{2}}{t}}}{|x-y|^{\gamma}} d y\right)^{(2-p) / 2 p}\left(\int_{\Delta_{j}} d y|W(y)|^{2}\right)^{1 / 2}= \\
\frac{t^{(p-1) / p}}{(2 \pi)^{1 / p}} \int_{\mathbf{R}^{3}} d x\left(\int_{\Delta_{0}} \frac{e^{-\gamma \frac{|x-y|^{2}}{t}}}{|x-y|^{\gamma}} d y\right)^{(2-p) / 2 p} \sum_{j \in \mathbf{Z}^{3}}\left(\int_{\Delta_{j}} d y|W(y)|^{2}\right)^{1 / 2},
\end{gathered}
$$

where $\gamma=2 /(2-p)$. Here $\Delta_{0}$ is the unit cube centered at the origin. Note that since $1<p<4 / 3$ we have $2<\gamma<3$. By means of the elementary estimate

$$
\int_{\Delta_{0}} \frac{e^{-\gamma \frac{|x-y|^{2}}{t}}}{|x-y|^{\gamma}} d y \leq C(\gamma) \frac{e^{-\gamma \frac{|x|^{2}}{2 t}}}{|x|^{\gamma}}
$$




\section{SCHRÖDINGER OPERATORS IN $R^{3}$ AND THE VAN HOVE LIMIT}

We start with a discusion of Schrödinger operators and the associated spectral shift functions in $\mathbf{R}^{3}$. As in the case of a bounded domain, $V \in \mathcal{K}$ is an infinitesimally small form bounded perturbation of $-\Delta$. The quadratic form of $H_{0}+V, H_{0}=-\Delta$ is closed on $\mathcal{Q}(-\Delta)$ and defines a semibounded selfadjoint operator $H$ [AiSi, Si2]. We recall that in the case of $\mathbf{R}^{3}$ we have the inclusion $l^{1}\left(L^{2}\right) \subset L^{1} \cap L^{2} \subset \mathcal{K}$. Due to this inclusion and Theorem C.7.8 of [Si2] we have that $\exp \{-t H\}-\exp \left\{-t H_{0}\right\}$ is trace class for any $t>0$. Therefore the spectral shift function

$$
\xi_{t}(\mu)=\xi\left(\mu ; e^{-t H}, e^{-t H_{0}}\right), \mu \in \mathbf{R}
$$

is defined by Krein's theorem [Kr1]. More precisely,

$$
\xi_{t}(\mu)=\pi^{-1} \lim _{\epsilon \rightarrow+0} \arg \operatorname{det}\left\{I+\left(e^{-t H}-e^{-t H_{0}}\right)\left(e^{-t H_{0}}-\mu-i \epsilon\right)^{-1}\right\}
$$

exists for almost all $\mu \in \mathbf{R}$. Note that

$$
\arg \operatorname{det}\left\{I+\left(e^{-t H}-e^{-t H_{0}}\right)\left(e^{-t H_{0}}-\mu-i \epsilon\right)^{-1}\right\}
$$

is well defined by the condition that it should tend to zero as $\epsilon \rightarrow+\infty$. The function $\xi_{t}$ is real valued and belongs to $L^{1}(\mathbf{R})$ with $\xi_{t}(\mu)=0$ for $\mu$ above the spectra of $e^{-t H}$ and $e^{-t H_{0}}$. Similar to Lemma 3.1 of [JeKa] it can be shown that $\xi(\lambda)=-\xi_{t}\left(e^{-\lambda t}\right)$ is the spectral shift function for the pair of the Hamiltonians $H$ and $H_{0}$,

$$
\xi\left(\lambda ; H, H_{0}\right)=-\xi_{t}\left(e^{-\lambda t}\right)
$$

such that

$$
\operatorname{tr}\left(\phi(H)-\phi\left(H_{0}\right)\right)=\int_{\mathbf{R}} \phi^{\prime}(\lambda) \xi\left(\lambda ; H, H_{0}\right) d \lambda
$$

for arbitrary $\phi \in C_{0}^{\infty}(\mathbf{R})$. We note that it does not depend on $t>0$ and $\xi\left(\lambda ; H, H_{0}\right)=0$ for $\lambda$ below the spectra of $H$ and $H_{0}$. 
when $x \in \Lambda$, and $G_{\Lambda}(x ; V, W)=0$ otherwise. Then, by the Feynman-Kac formula we get

$$
\begin{array}{r}
\operatorname{tr}\left(\exp \left\{-t\left(H_{0, \Lambda}+V\right)\right\}-\exp \left\{-t\left(H_{0, \Lambda}+V_{-}\right)\right\}\right)=\int_{\Lambda} d x G_{\Lambda}\left(x ; V, V_{-}\right), \\
\operatorname{tr}\left(\exp \left\{-t\left(H_{0, \Lambda}+V_{-}\right)\right\}-\exp \left\{-t H_{0, \Lambda}\right\}\right)=\int_{\Lambda} d x G_{\Lambda}\left(x ; V_{-}, 0\right) .
\end{array}
$$

We note that for given value of $t>0$,

$$
\begin{aligned}
& G_{\Lambda}\left(x ; V, V_{-}\right) \rightarrow \\
& (4 \pi t)^{-3 / 2} \mathbf{E}_{0, x}^{t, x}\left\{\exp \left\{-\int_{0}^{t} V\left(X_{u}\right) d u\right\}-\exp \left\{-\int_{0}^{t} V_{-}\left(X_{u}\right) d u\right\}\right\}, \\
& G_{\Lambda}\left(x ; V_{-}, 0\right) \rightarrow \\
& (4 \pi t)^{-3 / 2} \mathbf{E}_{0, x}^{t, x}\left\{\exp \left\{-\int_{0}^{t} V_{-}\left(X_{u}\right) d u\right\}-1\right\}
\end{aligned}
$$

when $\Lambda \rightarrow \infty$ for almost every $x \in \mathbf{R}^{3}$. Moreover,

$$
\begin{array}{r}
\left|G_{\Lambda}\left(x ; V, V_{-}\right)\right| \leq G^{(1)}(x)= \\
\mathbf{E}_{0, x}^{t, x}\left\{\exp \left\{-\int_{0}^{t} V_{-}\left(X_{u}\right) d u\right\}-\exp \left\{-\int_{0}^{t} V\left(X_{u}\right) d u\right\}\right\}, \\
\left|G_{\Lambda}\left(x ; V_{-}, 0\right)\right| \leq G^{(2)}(x)= \\
\mathbf{E}_{0, x}^{t, x}\left\{\exp \left\{-\int_{0}^{t} V_{-}\left(X_{u}\right) d u\right\}-1\right\} .
\end{array}
$$

Obviously, $G^{(i)}(x) \geq 0, G^{(i)} \in L^{1}\left(\mathbf{R}^{3}\right), i=1,2$. Thus, by the dominated convergence theorem we get

$$
\begin{array}{r}
\lim _{\Lambda \rightarrow \infty} G_{\Lambda}\left(x ; V, V_{-}\right)=-(4 \pi t)^{-3 / 2} \int_{\mathbf{R}^{3}} d x G^{(1)}(x), \\
\lim _{\Lambda \rightarrow \infty} G_{\Lambda}\left(x ; V_{-}, 0\right)=(4 \pi t)^{-3 / 2} \int_{\mathbf{R}^{3}} d x G^{(2)}(x),
\end{array}
$$

from which the claim of the lemma follows.

Denote

$$
\zeta\left(\lambda ; H_{0}+V, H_{0}\right)=\int_{-\infty}^{\lambda} \xi\left(\lambda^{\prime} ; H_{0}+V, H_{0}\right) d \lambda^{\prime}
$$


Therefore

$$
\begin{array}{r}
\zeta\left(\lambda ; H_{0, \Lambda}+V_{-}, H_{0, \Lambda}\right)=\int_{-\infty}^{\lambda} \xi\left(\lambda^{\prime} ; H_{0, \Lambda}+V_{-}, H_{0, \Lambda}\right) d \lambda^{\prime} \\
\zeta\left(\lambda ; H_{0, \Lambda}+V, H_{0, \Lambda}+V_{-}\right)=\int_{-\infty}^{\lambda} \xi\left(\lambda^{\prime} ; H_{0, \Lambda}+V, H_{0, \Lambda}+V_{-}\right) d \lambda^{\prime}
\end{array}
$$

are monotonic functions with respect to $\lambda$. Since $H_{0, \Lambda}, H_{0, \Lambda}+V_{-}$and $H_{0, \Lambda}+V$ are bounded from below uniformly in $\Lambda$ there is $b \leq 0$ not depending on $\Lambda$ such that $\zeta\left(\lambda ; H_{0, \Lambda}+V_{-}, H_{0, \Lambda}\right)$ and $\zeta\left(\lambda ; H_{0, \Lambda}+V, H_{0, \Lambda}+V\right)$ are identically zero for all $\lambda<b$. By these facts and Lemma 2.4 the condition (i) of Lemma 3.4 is satisfied for (3.6) and (3.7). Moreover, by Lemmas 2.4 and 3.2 the conditions (iii) and (iv) are also satisfied. Thus, by Lemma 3.4 the limits

$$
\begin{array}{r}
\lim _{\Lambda \rightarrow \infty} \zeta\left(\lambda ; H_{0, \Lambda}+V_{-}, H_{0, \Lambda}\right) \equiv \zeta_{\infty}\left(\lambda ; H_{0}+V_{-}, H_{0}\right), \\
\lim _{\Lambda \rightarrow \infty} \zeta\left(\lambda ; H_{0, \Lambda}+V, H_{0, \Lambda}+V_{-}\right) \equiv \zeta_{\infty}\left(\lambda ; H_{0}+V, H_{0}+V_{-}\right)
\end{array}
$$

exist and satisfy

$$
\begin{array}{r}
\operatorname{tr}\left(\exp \left\{-t\left(H_{0}+V_{-}\right)\right\}-\exp \left\{-t H_{0}\right\}\right)= \\
-t \int_{\mathbf{R}} e^{-\lambda t} d \zeta_{\infty}\left(\lambda ; H_{0}+V_{-}, H_{0}\right) \\
\operatorname{tr}\left(\exp \left\{-t\left(H_{0}+V\right)\right\}-\exp \left\{-t H_{0}+V_{-}\right\}\right)= \\
-t \int_{\mathbf{R}} e^{-\lambda t} d \zeta_{\infty}\left(\lambda ; H_{0}+V, H_{0}+V_{-}\right) .
\end{array}
$$

By Lemma 3.2, relation (3.4) and the uniqueness of the Laplace transform we get that

$$
\begin{array}{r}
\zeta\left(\lambda ; H_{0}+V_{-}, H_{0}\right)=\zeta_{\infty}\left(\lambda ; H_{0}+V_{-}, H_{0}\right), \\
\zeta\left(\lambda ; H_{0}+V, H_{0}+V_{-}\right)=\zeta_{\infty}\left(\lambda ; H_{0}+V, H_{0}+V_{-}\right),
\end{array}
$$

where the integrated spectral shift functions on the 1.h.s. are defined by (3.5). Applying the statement (i) of Lemma 3.1 completes the proof of the theorem. 


\section{APPENDIX}

Here we present the example of Hamiltonians with point interactions, formally given by potentials of the form

$$
V_{i}(x)=\mu \delta\left(x-a_{i}\right), i=1,2,
$$

where $a_{i} \in \mathbf{R}^{3}, a_{1} \neq a_{2}$, and for which the inequality (1.9) does not hold. Schrödinger operators corresponding to such interactions can be correctly defined and their resolvents can be calculated explicitly (see e.g. [AlGeH $\varnothing \mathrm{Ho}]$ ). In particular,

$$
R_{i}\left(x, x^{\prime} ; z\right)=R_{0}\left(x, x^{\prime} ; z\right)+\frac{1}{\left(4 \pi^{2}\right)^{2}}\left(\alpha-\frac{i \sqrt{z}}{4 \pi}\right)^{-1} \frac{e^{i \sqrt{z}\left|x-a_{i}\right|}}{\left|x-a_{i}\right|} \frac{e^{i \sqrt{z}\left|x^{\prime}-a_{i}\right|}}{\left|x^{\prime}-a_{i}\right|},
$$

where $R_{i}\left(x, x^{\prime} ; z\right)$ stands for the integral kernel of the resolvent $R_{i}(z)=$ $\left(H_{0}+V_{i}-z\right)^{-1}$ (formally), and $\alpha \in(-\infty,+\infty]$ is a parameter that can be related to the scattering length and serves to describe the strength of the point interaction thus replacing the formal coupling constant $\mu$ in (A.1). The limit $\alpha \rightarrow+\infty$ leads to the case of the free dynamics, i.e. $R_{i}(z) \rightarrow R_{0}(z)$ in norm for any $z$ with $\operatorname{Im} z \neq 0$ as $\alpha \rightarrow+\infty$. Obviously, we have

$$
\operatorname{tr}\left(R_{i}(z)-R_{0}(z)\right)=-\frac{1}{8 \pi i \sqrt{z}}\left(\alpha-\frac{i \sqrt{z}}{4 \pi}\right)^{-1}, \operatorname{Im} z \neq 0 .
$$

Here the root $\sqrt{z}$ is defined such that $\operatorname{Im} \sqrt{z}>0$ for $\operatorname{Im} z \neq 0$. From (A.2) and from the relation

$$
\operatorname{tr}\left(R_{i}(z)-R_{0}(z)\right)=-\int_{-\infty}^{\infty} \frac{\xi\left(\lambda ; H_{0}+V_{i}, H_{0}\right)}{(\lambda-z)^{2}} d \lambda
$$

it follows that

$$
\frac{d}{d \lambda} \xi\left(\lambda ; H_{0}+V_{i}, H_{0}\right)=-\frac{\alpha}{8 \pi^{2} \sqrt{\lambda}}\left(\alpha^{2}+\frac{\lambda}{(4 \pi)^{2}}\right)^{-1}, \lambda>0 .
$$

Taking into account that $H_{0}+V_{i}$ has the only eigenvalue $\lambda_{1}(\alpha)=-(4 \pi \alpha)^{2}$ when $\alpha<0$ and no eigenvalues when $\alpha \geq 0$ we get

$$
\xi\left(\lambda ; H_{0}+V_{i}, H_{0}\right)=-\theta(-\alpha) \theta\left(\lambda-\lambda_{1}(\alpha)\right)-\theta(\lambda) \frac{1}{\pi} \arctan \frac{\sqrt{\lambda}}{4 \pi \alpha},
$$




$$
\begin{array}{r}
-N(\alpha)-\frac{1}{\pi} \arctan \left\{\left[8 \pi \alpha \sqrt{\lambda}+\frac{\sin \left(2 \sqrt{\lambda}\left|a_{1}-a_{2}\right|\right)}{\left|a_{1}-a_{2}\right|}\right] .\right. \\
\left.\cdot\left[(4 \pi \alpha)^{2}-\lambda+\frac{\cos \left(2 \sqrt{\lambda}\left|a_{1}-a_{2}\right|\right)}{\left|a_{1}-a_{2}\right|}\right]^{-1}\right\}, \lambda \geq 0,
\end{array}
$$

where $N(\alpha)$ is a number of eigenvalues of the Hamiltonian $H_{0}+V_{1}+V_{2}$.

We note that the Hamiltonian $H_{0}+V_{1}+V_{2}$ has at most two (negative) eigenvalues counting multiplicity. They are determined by the equation $A(z)=0$. Now let $\alpha, a_{1}$, and $a_{2}$ be such that $0>4 \pi \alpha\left|a_{1}-a_{2}\right| \geq-1$. Then $H_{0}+V_{1}+V_{2}$ has the only simple eigenvalue $\lambda(\alpha)<0$. In this case, therefore, the spectral shift function for negative $\lambda$ is given by

$$
\xi\left(\lambda ; H_{0}+V_{1}+V_{2}, H_{0}\right)=-\theta(-\alpha) \theta(\lambda-\lambda(\alpha)), \lambda<0 .
$$

It is easy to see that the inequality (1.9) for $\lambda<0$ in the present context is equivalent to

$$
(\lambda(\alpha)-\lambda)_{-} \leq 2\left(\lambda_{1}(\alpha)-\lambda\right)_{-},
$$

where $(x)_{-}=x \theta(-x)$. It may be easily shown that for some values of $\alpha<0$ and $\lambda \leq 0$ the inequality (A.5) does not hold. In particular,

$$
\lambda(\alpha)>2 \lambda_{1}(\alpha)
$$

when $4 \pi \alpha\left|a_{1}-a_{2}\right| \in[-1,-c)$, with $c=0.790 \ldots$ being the solution of the equation

$$
(3-2 \sqrt{2}) x^{2}=e^{-2 \sqrt{2} x} .
$$




\section{References}

[AiSi] M. Aizenman and B. Simon, "Brownian motion and Harnack inequality", Commun. Pure Appl. Math. 35, 209-273 (1982)

[AlAr] S. Albeverio and T. Areda, "The relation between quantum mechanics and classical mechanics: a survey of some mathematical aspects", pp. 37-76 in Chaotic Behavior in Quantum Systems (G.Casati, ed.) NATO ASI Series, Vol. 120. Plenum, New York, 1985.

[AlGeHøHo] S. Albeverio, F. Gesztesy, R. Høegh-Krohn, and H. Holden, Solvable Models in Quantum Mechanics. Springer, 1988.

[BaBl] R. Balian and C. Bloch, "Solution of the Schrödinger equation in terms of classical paths", Ann. Phys. 85, 514-545 (1974)

[BiKr] M.Sh. Birman and M.G. Krein, "On the theory of wave operators and scattering operators", Sov. Math. - Doklady 3, 740-744 (1962)

[BiSol] M.Sh. Birman and M.Z. Solomjak, "Estimates of singular numbers of integral operators. III. Operators in unbounded domains", Vestnik Leningrad. Univ. 24, 35-48 (1969) (in Russian)

[BiSo2] M.Sh. Birman and M.Z. Solomjak, "Asymptotic behavior of the spectrum of differential equations", J. Sov. Math. 12, 247-283 (1979)

[BiYa] M.Sh. Birman and D.R. Yafaev, "The spectral shift function. Works by M.G.Krein and their development", Algebra i Analis 4, 1-44 (1992) (in Russian)

[Bu] V.S. Buslaev, "The trace formulae and certain asymptotic estimates of the kernel of the resolvent for the Schrödinger operator in threedimensional space", pp. 69-85 in Topics in Mathematical Physics, Vol.1, (M.Sh. Birman, ed.) Plenum, New York, 1967.

[CaLa] R. Carmona and J. Lacroix, Spectral Theory of Random Schrödinger Operators, Birkhäuser, Boston, Basel, Berlin, 1990. 
[McKSi] H.P. McKean and I.M. Singer, "Curvature and the eigenvalues of the Laplacian", J. Diff. Geom. 1, 43-69 (1967)

[Mo] S.A. Molchanov, "Diffusion processes and Riemannian geometry", Russian Math. Surveys 30:1, 1-63 (1975)

[Pa] L.A. Pastur, "On the Scrödinger equation with a random potential", Theor. Math. Phys. 6, 299-306 (1971)

[RS4] M. Reed and B. Simon, Methods of Modern Mathematical Physics, Vol.4: Analysis of Operators. Academic Press, New York, 1978.

[Ro] D. Robert, "Asymptotique de la phase de diffusion a haute energie pour des perturbations du Laplacien", Seminaire sur les equations aux derivees partielles, Ecole Polytechnique, Exposé n XVII (1989)

[Ru] D. Ruelle, Statistical Mechanics. Benjamin, New York, 1969.

[Sch] R. Schrader, "High energy behavior for non-relativistic scattering by stationary external metrics and Yang-Mills potentials", Z. Physik C 4, 27-36 (1980)

[Si1] B. Simon, Functional Integration and Quantum Physics, Academic Press, New York, 1979

[Si2] B. Simon, "Schrödinger semigroups", Bull. AMS, 7, 447-526 (1982)

[Th] W. Thirring, Lehrbuch der mathematischen Physik, Vol. III: Quantenmechanik von Atomen und Molekulen. Springer, Vienna, 1979.

[Wa] R.T. Waechter, "On hearing the shape of a drum: an extension to higher dimensions", Proc. Camb. Phil. Soc. 72, 439-447 (1972)

[Wi] D.V. Widder, The Laplace Transform. Princeton University Press, Princeton, 1946. 